\title{
Path Planning in the Presence of Obstacles Based on Task Requirements
}

\author{
Tzung-hsien Wu and Kuu-young Young* \\ Department of Control Engineering \\ National Chiao-Tung University \\ Hsinchu 30039, Taiwan, R.O.C. \\ Received January 31, 1992; revised April 5, 1993; \\ accepted May 2, 1994
}

\begin{abstract}
We propose a novel and efficient scheme for planning a kinematically feasible path in the presence of obstacles according to task requirements. By employing geometrical analysis, we derive expressions to describe the relationship between the planned path, kinematic constraints, and obstacles in the robot workspace. The freedom available according to task requirements is then utilized to modify the infeasible portions of the planned path. We use a $6 \mathrm{R}$ (revolute) wrist-partitioned type of robot manipulator and a spherical obstacle as a case study to demonstrate the proposed scheme. We then extend our results to general wrist-partitioned types of robot manipulators and arbitrarily-shaped or multiple obstacles. (c) 1994 John Wiley \& Sons, Inc.

ここでは、障害物が存在する中で、タスク要求に従って運動学的に適当なバスを計画する、斬新で効果的な方法 を提案する。幾何学的解析を探用し、ロボットの作業空間において、計画されたパス、運動学的束縛、障害物の 間の関係を表現する式を誘導する。夕スク要求に对して、利用可能な自由度は、計画されたパスの不適当な部分 の修正に使われる。そして、6R (nevolute)りスト・パーティッション型のロボット・マニピェレータと、球形の障 害物を実例研究として使い、提案した方法の特徽について説明する。さらに、今回の寒駼結果を、一般のリス ト・パーティッション型のロボット・マニピュレータと、暖昧な形や椱数の障害物に拡張して適応する。
\end{abstract}

\section{INTRODUCTION}

Robot trajectories are planned according to different industrial tasks. To obtain feasible trajectories, dif-

*To whom all correspondence should be addressed. ferent constraints need to be satisfied, including task specifications, the avoidance of obstacles within the robot workspace, and the inherent limitations of the robot manipulator. These constraints are summarized in Table I. The task-level constraint arises 
Table 1. Constraints for a feasible trajectory.

\begin{tabular}{ll}
\hline Constraint & \multicolumn{1}{c}{ Attributes } \\
\hline Task-level & Position, orientation specifications, etc. \\
Geometric & Obstacles \\
Kinematic & Joint types, ranges, and limits \\
Dynamic & Velocity, acceleration limits, etc. \\
\hline
\end{tabular}

from different task requirements. For instance, if the task is to move a directionless object from one location to another, then only the starting and end positions need to be considered, and the intermediate positions and orientations may be arbitrary. On the other hand, if the object to be moved is a cup of coffee, then the orientation specification also needs to be taken into account. The constraint due to the presence of obstacles inside the robot workspace, which is usually identified as the geometrical constraint, is met by planning collision-free paths among obstacles. ${ }^{1}$ The kinematic constraint is due to joint types, ranges of motion, and other kinematic limitations of the robot manipulator used. Last, the dynamic constraint results from the velocity and acceleration limitations of the robot actuators. Planning robot trajectories to meet these constraints generally involves two steps: first, we find a feasible path that meets the task-level, geometric, and kinematic constraints; second, we design a velocity profile along the planned path that satisfies the dynamic constraints. ${ }^{2}$

The discussion in this article will focus on the first of the two stages just mentioned. There are several common approaches to planning a feasible path. One is to plan a path in Cartesian space by specifying both position and orientation for each point on the path. ${ }^{3,4}$ The rationale for this approach is that both position and orientation need to be specified to find unique corresponding joint positions. The path planned by this approach tends to induce unnecessary infeasibility because extra constraints are imposed. Moreover, because knowledge of the kinematic constraints is not incorporated in the planning, kinematically infeasible paths cannot be modified and must be replanned. Even after replanning, the procedure is still one of trial-and-error, for the replanned path may still be infeasible.

Another approach to planning a feasible path is to search for a feasible path in the joint or configuration space according to the kinematic constraint or obstacles. A famous method is the configuration space (C-space) approach. ${ }^{5}$ In the configuration space approach, obstacles for a manipulator with $n$ joints are formulated as C-space obstacles of $n$-dimensional volume. To represent an $n$-dimensional $\mathrm{C}$-space obstacle in C-space, the obstacle is recursively sliced into obstacles of fewer dimensions, until a union of one-dimensional volumes is obtained. Feasible paths are then located by searching the free regions in the configuration space to avoid obstacles and constraints from the kinematics. The main problem with this approach is the complexity of the C-space obstacle, especially when the dimensionality of the C-space is high. To reduce the complexity of the C-space obstacle, in ref. 6 a sequential search strategy was developed based on the assumption that the motion of a proximal link does not depend on the motion of a distal link. One $n$ dimensional problem for an $n$-link manipulator arm is reduced to an $n-1$ two-dimensional planning problem. However, this approach may not find a collision-free path in some cluttered environments even if one such path exists. In addition, mapping the task-level constraints in Cartesian space onto joint or configuration space is by no means an easy task.

We propose a novel and efficient approach, based on task requirements, to designing a kinematically feasible path in the presence of obstacles. Instead of searching for a feasible path in the configuration space, as in ref. 5 , we propose first to plan a path in a way similar to the approach in refs. 3 and 4 , by specifying both position and orientation for each point on the path. Then, according to different task requirements, we will modify the portions of the path that are infeasible due to kinematic constraints and obstacles. As a result, a feasible path satisfying the task-level, geometrical, and kinematic constraints can be found in a smaller search space. To ensure that the modification part of our approach will succeed, we also develop representations of kinematic constraints and obstacles in the robot workspace and appropriate modification strategies.

We use a geometrical approach to derive expressions that accurately describes the relationship between the robot workspace and the planned path. ${ }^{7,8}$ Through this analysis, different geometrical regions corresponding to the kinematic constraints of the robot manipulator used and obstacles will be identified in the robot workspace. For details on how to generate regions corresponding to kinematic constraints inside the robot workspace, refer to ref. 9. A classification of modification strategies corresponding to various task requirements can also be found in ref. 9. This article will concentrate on how obsta- 
cles inside the robot workspace are to be formulated and how modification strategies can be developed to cope with the presence of obstacles. We use the six revolute $(R)$ joint wrist-partitioned type of robot manipulator shown in Figure 1 and a spherical obstacle as a case study. We then extend our results to general wrist-partitioned types of robot manipulators and arbitrarily-shaped or multiple obstacles. Simulations are performed to demonstrate the proposed schemes.

\section{WORKSPACE ANALYSIS}

The workspace of a robot manipulator can be separated into two parts: the positional and orientational workspaces. A planned path is feasible if points on the path are within the feasible regions of these two workspaces. To take advantage of the wrist-partitioned structure of the $6 R$ non-redundant robot manipulator used in this study, we will select the wrist position $p_{w}$ as a reference point. Therefore the workspace can be divided into the workspace of the wrist and that of the minor joints. There will then be two 3D (3-dimensional) workspaces to be analyzed instead of a $6 \mathrm{D}$ workspace. Consequently, 3D obstacles can be easily formulated inside the workspaces.

The positional workspace can be defined as that of the wrist constrained by the primary joints, denoted by $P W K$. Inside $P W K$, geometrical regions corresponding to different kinematic constraints, such as feasible regions, areas of different configurations, and singular areas, can be determined. Here the feasible regions correspond to the joint variables within the joint ranges, the areas of different configurations are defined as different sets of points with

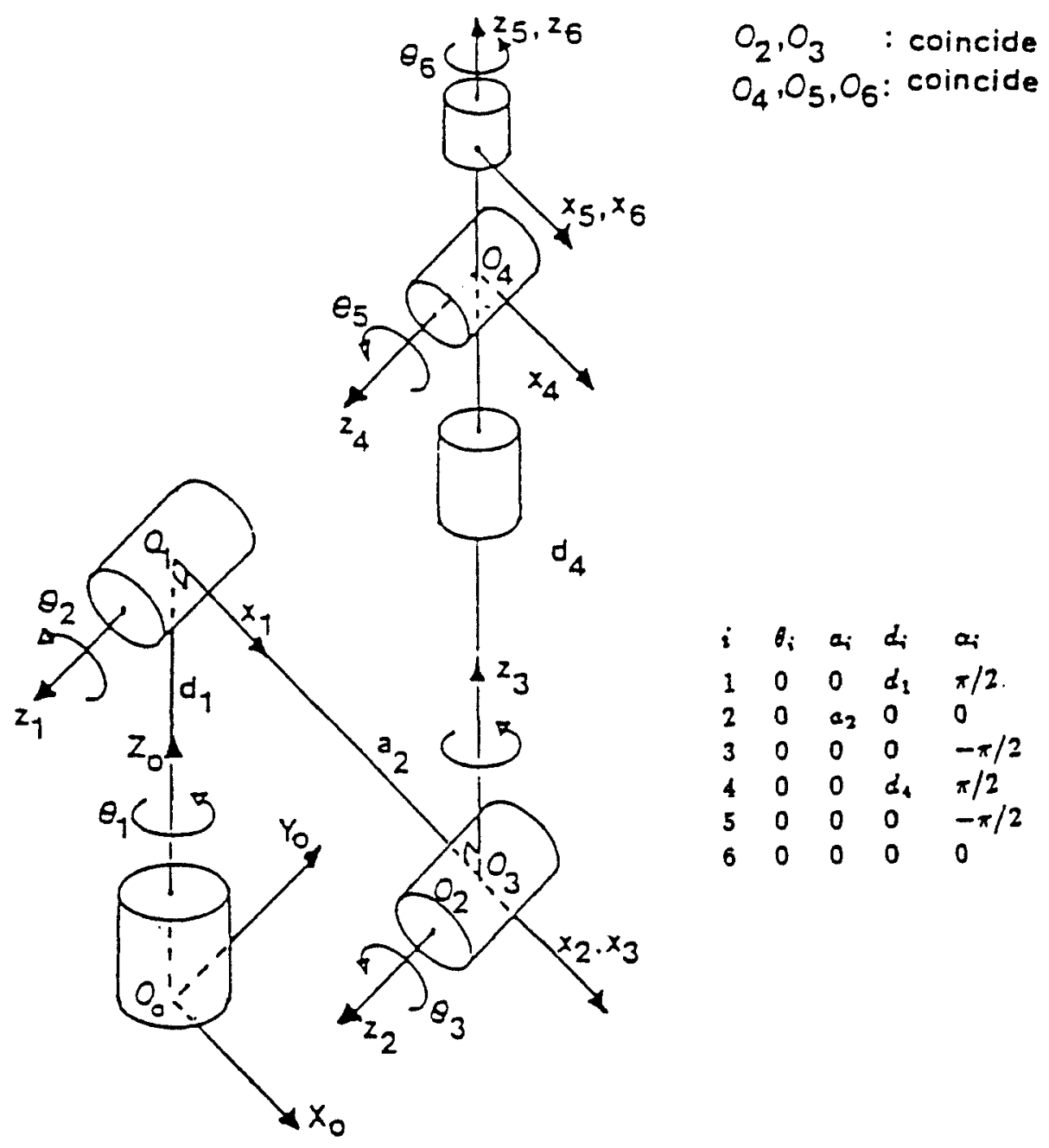

Figure 1. $6 \mathrm{R}$ robot manipulator. 
the same arm configuration, and the singular areas are the vicinities of the boundaries of the feasible regions and positions where the corresponding joint variables are undefined. ${ }^{10}$ With these geometrical regions described in the robot workspace, the planned path can also be identified inside the workspace by mapping the sets of joint variables specifying the planned path onto corresponding traces in the regions. Using this geometrical approach, the mapping transforms the analysis of joint variables, represented numerically, into traces in the workspaces, represented geometrically. As a result, geometric information related to kinematic constraints, obstacles, and neighboring points on the path in these workspaces can be derived.

It can be seen that each element within PWK will not only determine a wrist position but also a wrist coordinate frame for mounting the minor joints. Thus the orientational workspace, denoted by $O W K$, consists of two parts: the orientational workspace of the wrist as a function of the primary joints, denoted by $O W K^{p}$, and the orientational workspace as a function of the minor joints, denoted by $O W K^{m}$. The feasible regions, areas of different configurations, and singular areas can all be identified within $O W^{m}$.

\subsection{Positional Workspace}

To identify $O W K^{m}$ with three degrees of freedom (dof), we need to mount an end-effector with lengths in at least two different orientations upon the wrist. ${ }^{9}$ Without loss of generality, we may specify the end-effector to consist of two lengths, $h_{z}$ in the $\underline{a}_{e}$ direction and $h_{y}$ in the $\underline{o}_{e}$ direction. Using the lengths defined for the end-effector, we can derive the wrist position $p_{w}$ for analyzing $P W K$ from the end-effector's location, POS, as follows:

$$
p_{w}=\underline{p}_{e}-h_{y} \underline{Q}_{e}-h_{z} \underline{a}_{e}
$$

where $\underline{p}_{e}$ and $\left[\underline{n}_{e}, \underline{o}_{e}, \underline{a}_{e}\right]$ represent the position and orientation of $P O S$, respectively.

Because the traces of links two and three due to the rotations of joints two and three are in the same plane in this case study, we need to further divide the analysis of $P W K$ into two subworkspaces: $P W K_{1}$, corresponding to joint one, and $P W K_{23}$, corresponding to joints two and three.

\subsection{1. $P W K_{1}$ in the Presence of an Obstacle}

The spherical obstacle, denoted by $O B S$, is specified to be of radius $r$, with its center at $\left(x_{r}, y_{r}, z_{r}\right) . P W K_{1}$

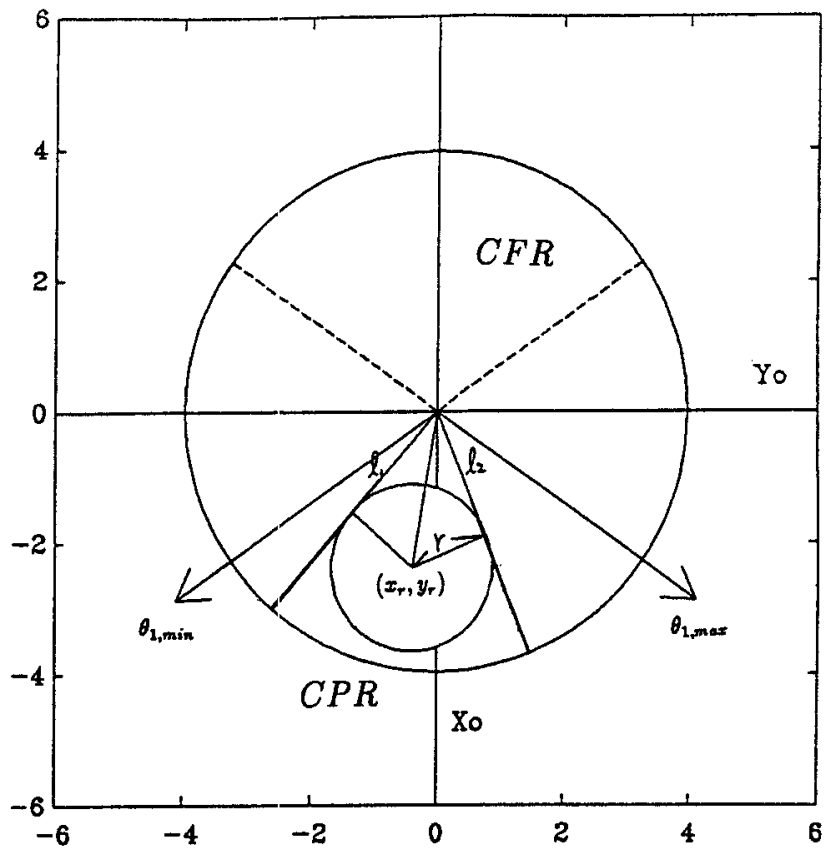

Figure 2. Workspace of joint one with obstacle.

is divided into four regions of different configurations by the lines corresponding to projections of $p_{w}$ when joint one is at the maximum $\left(\theta_{1, \max }\right)$ and minimum $\left(\theta_{1, \min }\right)$ positions, respectively. ${ }^{9}$ By projecting $O B S$ onto the $\left(x_{0}, y_{0}\right)$ plane, we can obtain $P W K_{1}$ in the presence of $O B S$, as shown in Figure 2. Projections of $O B S$ form a circle, $O B S_{1}$, with radius $r$ and its center at $\left(x_{r}, y_{r}\right)$. The slopes $\left(\tan \theta_{1}\right)$ of the two lines tangential to $O B S_{1}, \ell_{1}$ and $\ell_{2}$, can be determined by the following equation:

$$
\left|\frac{y_{r}-\tan \theta_{1} x_{r}}{\sqrt{1+\tan ^{2} \theta_{1}}}\right|=r .
$$

Using $\ell_{1}$ and $\ell_{2}$, we can separate $P W K_{1}$ into a collision free region (CFR) and collision possible region $(C P R)$, corresponding to the regions without and with $O B S_{1}$, respectively. $O B S$ will not affect the feasibility of the projections of the wrist path within the $C F R$. However, for projections within the CPR, it is possible that the wrist may collide with the obstacle, depending upon the feasibility analysis in $P W K_{23}$.

\subsection{2. $P W K_{23}$ in the Presence of an Obstacle}

To generate $P W K_{23}$, we derive ${ }^{1} p_{w}\left({ }^{1} x_{w},{ }^{1} y_{w},{ }^{1} z_{w}\right)$ by removing from $p_{w}$ the effect due to the rotation of joint one. Then $P W K_{23}$ can be obtained by rotating joints two and three from their minimums to maxi- 
mums. Due to the geometries of joints two and three, $P W K_{23}$ depends not only on the limits of $\theta_{3}$ but also on its range. It takes four cases and five circle equations to specify the boundaries of $P W K_{23} .{ }^{9}$

To analyze $P W K_{23}$ in the presence of $O B S$, we need to find the mapping of $O B S$ on $P W K_{23}$. We propose slicing $O B S$ by the planes containing the $z_{0}$ axis and rotating the slices back to the $\left(x_{1}, y_{1}\right)$ plane of $\theta_{1}=0$, where $P W K_{23}$ is located. Because $O B S$ is a sphere, the slices will contain circles with different radii. To avoid performing a separate analysis for each slice, we will choose the circle with the largest radius $r_{\max }, O B S_{23}$, which covers all the others, as the mapping of $O B S$ on $P W K_{23}$. The center of $O B S_{23}$ is defined as $\left({ }^{1} x_{r},{ }^{1} y_{r}\right)$. Choosing $O B S_{23}$ in this way means that we will be analyzing an obstacle that is larger than $O B S$. This expanded obstacle is donutshaped. It can be seen that if a donut-shaped obstacle with its circular side perpendicular to the $\left(x_{1}, y_{1}\right)$ plane is analyzed through the above procedure, it will turn out that we actually are not dealing with an expanded obstacle after all. This implies that a donut-shaped object may be a better representation of an obstacle than the spherical object used in this approach. A further discussion of obstacle representation is in section 3.2 .

Depending on the location of $O B S_{23}$ in $P W K_{23}$, the path of link two will fall into one of three cases: (a) Link two cannot reach $O B S_{23}$; (b) Link two can reach $O B S_{23}$ and is tangential to it when contact occurs; (c) Link two can reach $O B S_{23}$ and is not tangential to it when contact occurs. In the following, we use case (a) as an example for demonstration; the other two cases can be tackled in a manner similar to that used for case (a). Four circles, $C_{1}-C_{4}$, will be employed in the analysis of case (a) (see Fig. 3):

$$
\begin{aligned}
& C_{1}:{ }^{1} x_{w}^{2}+{ }^{1} y_{w}^{2}=a_{2}^{2}+d_{4}^{2}-2 a_{2} d_{4} \sin \theta_{3, \max } \\
& C_{2}:{ }^{1} x_{w}^{2}+{ }^{1} y_{w}^{2}=a_{2}^{2} \\
& C_{3}:{ }^{1} x_{w}^{2}+{ }^{1} y_{w}^{2}=a_{2}^{2}+d_{4}^{2}-2 a_{2} d_{4} \sin \theta_{3, \min } \\
& C_{4}:{ }^{1} x_{w}^{2}+{ }^{1} y_{w}^{2}=\left(a_{2}+d_{4}\right)^{2}
\end{aligned}
$$

where $C_{1}$ and $C_{3}$ represent the circles when joint two rotates throughout its range with joint three equal to $\theta_{3, \max }$ and $\theta_{3, \min }$, respectively; $C_{2}$ the circle with radius equal to the length of link two $a_{2}$; and $C_{4}$ the circle with radius $a_{2}+d_{4}$, specifying the farthest boundary of $P W K_{23}$. In Figure 3, the portion of $P W K_{23}$ shown is in the middle range of $\theta_{2}$, and it is assumed that $\left\|{ }^{1} p_{w}\right\|<a_{2}$ when $\theta_{3}=\theta_{3, \max }$ and $\left\|{ }^{1} p_{w}\right\|>$ $a_{2}$ when $\theta_{3}=\theta_{3, \min }$.

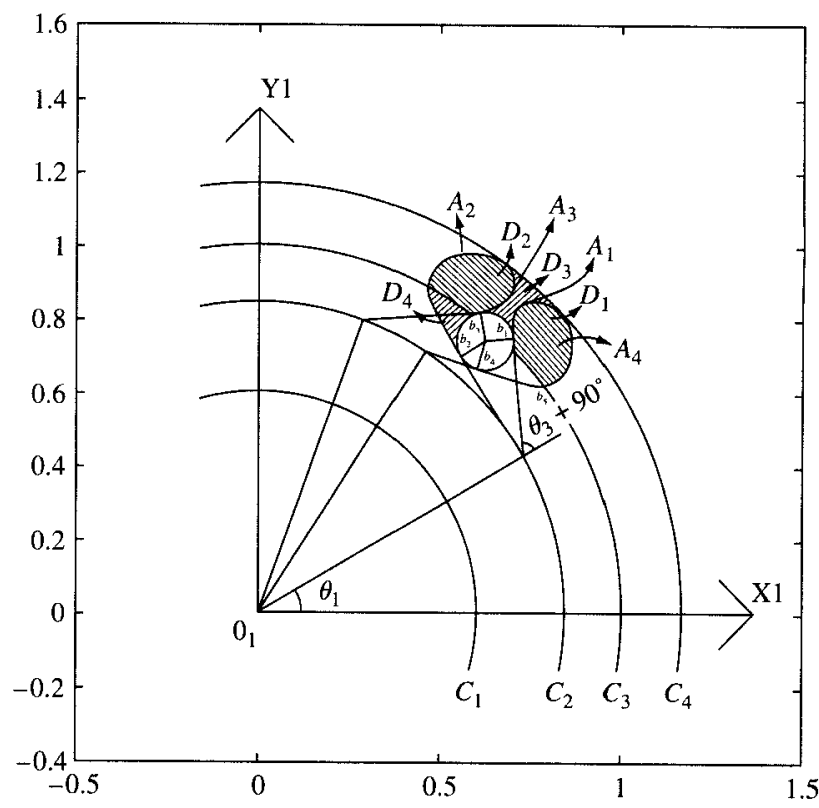

Figure 3. Workspace of joints two and three with obstacle.

There are two kinds of configurations in $P W K_{23}$, elbow-flip (EF), and elbow-nonflip (ENF). With $\theta_{3}$ specified to be $-90^{\circ}$ when links two and three are along the same line, the EF configuration is obtained when $\theta_{3} \in\left[\theta_{3, \min },-90^{\circ}\right]$, and ENF is obtained when $\theta_{3} \in\left[-90^{\circ}, \theta_{3, \max }\right]$. In Figure 3 , the region between $C_{3}$ and $C_{4}$ is relevant to both configurations, and that between $C_{1}$ and $C_{3}$ to the ENF configuration only. In the presence of $O B S_{23}$, the feasible region will be reduced by the area not only of $O B S_{23}$ but also its neighboring area.

To analyze how $P W K_{23}$ is affected by $O B S_{23}$, we employ the end positions of links two and three, $P_{1}\left(p_{1 x}, p_{1 y}\right)$ and $P_{2}\left(p_{2 x}, p_{2 y}\right)$. If we consider the ENF configuration in Figure 3 , curves $A_{1}$ and $A_{2}$ are the traces of $P_{2}$ when link three is tangential to $O B S_{23}$ from different sides. Curves $A_{1}$ and $A_{2}$ start from $b_{1}$ and $b_{2}$, where $P_{2}$ contacts $O B S_{23}$, and end when links two and three are aligned along the same line. The $\theta_{2}$ and $\theta_{3}$ corresponding to these curves can be obtained as follows:

$$
\begin{gathered}
\left|\frac{\left({ }^{1} y_{r}-p_{1 y}\right)-\tan \left(\theta_{2}+\theta_{3}+90^{\circ}\right)\left({ }^{1} x_{r}-p_{1 x}\right)}{\sqrt{1+\tan ^{2}\left(\theta_{2}+\theta_{3}+90^{\circ}\right)}}\right|=r_{\max }, \\
\theta_{2} \in\left[u_{1}, u_{2}\right]
\end{gathered}
$$

where $u_{1}$ corresponds to $\theta_{2}$ when $P_{2}$ contacts $O B S_{23}$ and $u_{2}$ to $\theta_{2}$ when links two and three are aligned along the same line. Similarly, if we consider the EF 
configuration, curves $A_{3}$ and $A_{4}$ can also be obtained, except that curve $A_{4}$ will start from $b_{5}$ instead of $b_{4}$, where $b_{5}$ is the intersection of the tangential curve and $C_{3}$. With curves $A_{1}-A_{4}$, in Figure 3 the area bounded by $A_{1}, A_{2}, C_{4}$, and $O B S_{23}$ cannot be reached by the ENF configuration, while the area bounded by $A_{3}, A_{4}, C_{3}, C_{4}$, and $O B S_{23}$ cannot be reached by the $\mathrm{EF}$ configuration. Based on the above analysis, four areas $D_{1}-D_{4}$ in $P W K_{23}$ affected by the presence of $O B S_{23}$ can be identified: $D_{1}$ becomes only relevant to the ENF configuration; $D_{2}$ becomes only relevant to the EF configuration; and $D_{3}$ and $D_{4}$ are not feasible now.

\subsection{Orientational Workspace}

To analyze OWK, we employ the orientation $R_{e}=$ $\left[\underline{n}_{e} \underline{\underline{o}}_{e} \underline{a}_{e}\right]$, which we represent as

$$
R_{e}=R_{w} *{ }^{w} R_{e} .
$$

As indicated in Eq. (8), $R_{w}$ serves as a reference coordinate frame for mounting ${ }^{w} R_{\ell}$. Because $R_{w}$ can be determined after the analysis of $p_{w}$ in $P W K$, the effect of $O W K^{p}$ can be removed and ${ }^{w} R_{e}$ can be solved from $R_{e} \cdot{ }^{9}$ Consequently, the analysis of OWK becomes that of $O W K^{m}$. With the end-effector position constrained by the limits and ranges of the minor joints, the analysis of $O W K^{m}$ is equivalent to the positional feasibility analysis of the end-effector. ${ }^{9}$

The end-effector can be represented in the wrist coordinates $\left(x_{w}, y_{w}, z_{w}\right)$ by the following two equations:

$$
\begin{aligned}
& { }^{w} \underline{h}_{y}=\left[\begin{array}{lll}
{ }^{w} h_{y_{x}} & { }^{w} h_{y_{y}} & { }^{w} h_{y_{z}}
\end{array}\right]^{t}=h_{y}{ }^{w} \underline{Q}_{e} \\
& { }^{w} \underline{h}_{z}=\left[\begin{array}{lll}
{ }^{w} h_{z_{x}} & { }^{w} h_{z_{y}} & { }^{w} h_{z_{z}}
\end{array}\right]^{t}=h_{z}{ }^{w} \underline{a}_{e}
\end{aligned}
$$

where ${ }^{w} \underline{n}_{e},{ }^{w} \underline{Q}_{e}$, and ${ }^{w} \underline{a}_{e}$ are the orientational vectors of ${ }^{w} R_{e}$. In Figure 1, the workspace of ${ }^{w} \underline{h}_{z}$ can be constrained only by the ranges and limits of joints four and five. Therefore ${ }^{w} h_{z}$ can be used to analyze the workspaces of joints four and five. Because the rotations of these two joints are not about the same axis, two subworkspaces are formed corresponding to joints four and five. The workspace swept by ${ }^{w} \underline{h}_{y}$, on the other hand, is constrained by all three joints; however, after the analyses of the workspaces of joints four and five, their effects can be removed and ${ }^{w} h_{y}$ used to analyze the workspace of joint six alone. ${ }^{9}$ In general, the end-effector is small compared with the links corresponding to the major joints. Therefore, to ensure that the presence of $O B S$ will not affect $O W K^{m}$, we propose expanding the radius of $O B S$ by all or a portion of the length of the end-effector, $\sqrt{h_{y}^{2}+h_{z}^{2}}$. The expansion of $O B S$ will depend on different modification strategies; we will return to this problem in section 4 .

\section{EXTENSION TO GENERAL CASES}

The analysis of the above case study can be extended to (a) general wrist-partitioned types of robot manipulators and (b) arbitrarily-shaped or multiple obstacles.

\subsection{General Wrist-Partitioned Types of Robot Manipulators}

To extend the results of the case study to general wrist-partitioned types of robot manipulators, we need to consider three major factors: offsets, prismatic joints, and the different connections between the joints. First, the effect of offsets can be identified as follows. For $P W K_{23}$ of the case study, for example, we assume that both offsets are present and that they are perpendicular and parallel to links two and three, respectively. If the traces of the links and offsets to the $\left(x_{1}, y_{1}\right)$ plane of $\theta_{1}=0^{\circ}$ are rotated about the $z_{0}$ axis and their projections are taken, the projections of the offset perpendicular to the links will have no effect on the traces. The projections of the offset parallel to the links are stationary with respect to the links and can be viewed as parts of the links. The above reasoning can also be applied to $P W K_{1}$. Thus, after the effect of the offsets is identified, the proposed approach can also be utilized to analyze robot manipulators with offsets.

Second, it can be seen that workspace analyses involving prismatic joints are much simpler than those involving revolute ones. This is because a prismatic joint rnoves the link along a straight line, while a revolute joint moves it along a curve. Third, there are three non-redundant ways in which three major points may be connected: (a) last two joints in parallel, (b) first two joints in parallel, and (c) all three joints consecutively perpendicular. The first case is the same as that of the case study. The analysis of the second case will be similar to case (a) if it is performed by starting from the third joint, and then going on to the first two joints.

As for the third case, the wrist position alone cannot uniquely determine the corresponding positions of the primary joints. More measured positions or orientations besides the wrist position are 
needed to analyze PWK. The connection of $O W K^{m}$ in the case study, on the other hand, is general, because the minor joints need to be consecutively perpendicular to be non-redundant.

\subsection{Arbitrarily-Shaped or Multiple Obstacles}

A spherical obstacle was used in the case study in this article. However, arbitrarily shaped or multiple obstacles may be present in an industrial process. To tackle an arbitrarily shaped obstacle utilizing our method, we divide and bound the obstacle by several spherical obstacles of equal or different radii. Figure 4 depicts an obstacle bounded by two spheres. The obstacle resulting from the dividing procedure should be the minimum expansion of the original one. When there are multiple spherical obstacles in the workspace, the resulting boundaries will be generated by merging the boundaries corresponding to each obstacle. Taking $P W K_{23}$ as an example, we use two circular $O B S_{23}$ in $P W K_{23}$ to demontrate how the boundaries of $D_{1}$ to $D_{4}$ are to be merged. There are three cases to consider: two obstacles (a) without intersections, (b) tangential to each other, and (c) overlapping. The merging result for case (a) is shown in Figure 5, and those for cases (b) and (c) can be obtained similarly.

Obstacle shapes other than a sphere can also be used as the basic element in constructing an irregularly shaped obstacle. The choice of which shape to

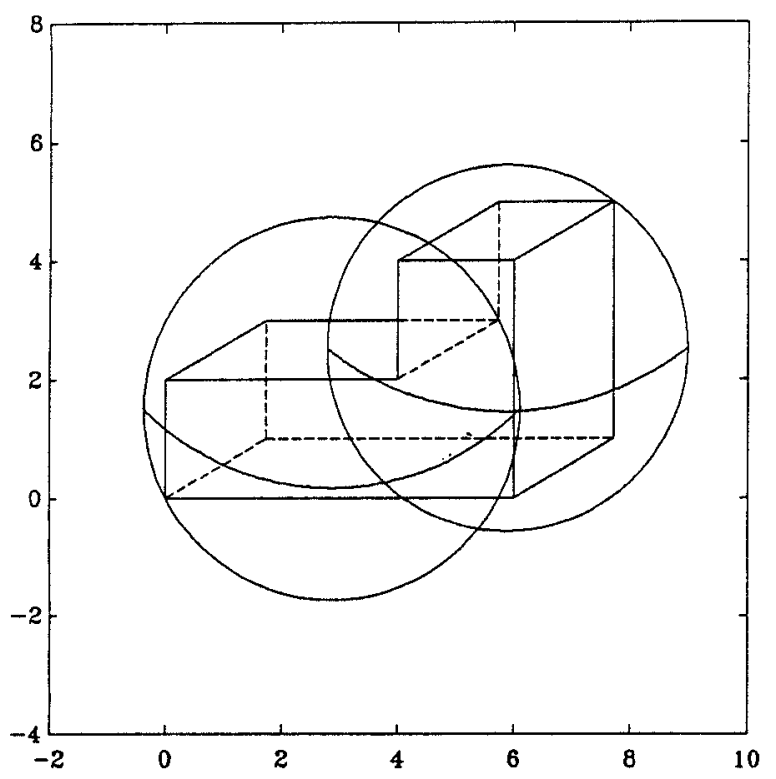

Figure 4. Irregularly-shaped obstacles represented by several spheres.

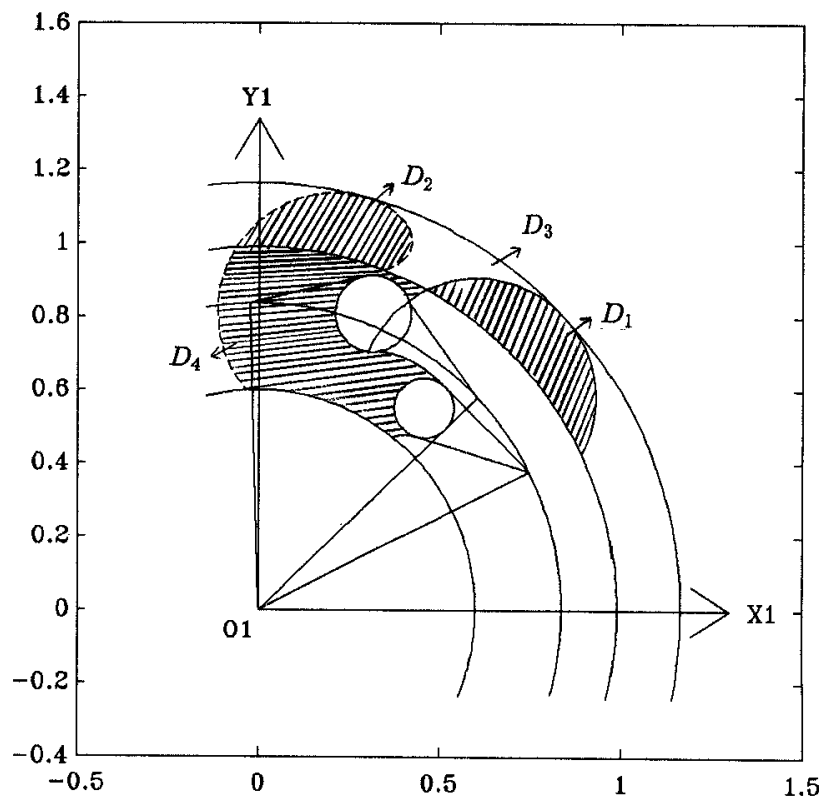

Figure 5. $P W K_{23}$ with two obstacles.

use involves a trade-off between a better representation for geometrical analysis and a better element for constructing the target obstacles. For instance, unlike a spherical obstacle, when a donut-shaped obstacle is placed with its circular side perpendicular to the $\left(x_{1}, y_{1}\right)$ plane, the analysis in $P W K_{23}$ will not require dealing with an expanded obstacle, as in the discussion in section 2.1.2. This indicates that a donut-shaped object may be a better representation for use with our method. However, when an irregularly shaped obstacle is constructed using several donut-shaped obstacles with certain orientations, many of the feasible regions may be discarded as a result. The choice of the basic element aforementioned will depend on the shapes of the obstacles encountered during various tasks.

\section{PATH PLANNING AND MODIFICATION}

Through the above analyses of $P W K$ and OWK, geometric information about a planned path and the workspaces can be derived for use in modifying infeasible portions of the planned path in the workspace. When no obstacle is involved, feasible paths can always be found between two feasible points due to the continuity of the workspace. This statement is no longer true, however, when certain constraints due to task requirements and obstacles are imposed. Therefore, the freedom available for modification will depend on task requirements, and ap- 
propriate modification strategies will vary accordingly. ${ }^{9}$ With the derived geometrical information and appropriate modification strategies, the path planning scheme described in the introduction can be utilized to design a kinematically feasible path in the presence of obstacles based on task requirements. The algorithm for this scheme is as follows:

Path Planning Algorithm: Plan a feasible path satisfying task-level, geometrical, and kinematic constraints.

Step 1: Design a path by specifying both position and orientation for each point on the path.

Step 2: Analyze the robot workspace using the proposed geometrical approach and construct subworkspaces corresponding to kinematic constraints, such as feasible regions, areas of different configurations, and singular areas.

Step 3: Formulate obstacles inside the workspace constructed in step 2 .

Step 4: Derive geometrical information about the planned path and the robot workspace described in step 3 . Then apply appropriate modification strategies based on task requirements to modify the infeasible portions of the planned path.

\subsection{Path Modification}

To accomplish the modification part of step 4 of the path planning algorithm, we need appropriate modification strategies for cases where obstacles are present. For tasks with the position (orientation) limitation, the dof in the orientation (position) can be utilized for modification. ${ }^{9}$ If there are no constraints for the given task, then the dof in both position and orientation can be used for modification. This situation can be taken to be an obstacle avoidance problem in the workspace by treating both obstacles and infeasible regions due to kinematic constraints as obstacles. In cases where both position and orientation specifications have to be satisfied, only feasibility can be checked, because there are no dof for modification. We will attempt, then, to develop algorithms for modifying an infeasible path in the presence of obstacles by utilizing the dof in position and orientation, respectively.

\subsubsection{Position Modification in the Presence of Obstacles}

Position modification is intended for cases with the orientation limitation; thus we will use the dof in position for modification. In general, the length of the end-effector is small compared with the links corresponding to the major joints. The proposed scheme for position modification will enlarge the radius of the obstacle by the length of the end-effector. Hence $O W K^{m}$ will not be affected by the obstacle. Because the joint ranges for the minor joints of most industrial robots are quite large, $O W K^{m}$ is usually kinematically feasible. For instance, the PUMA 560 has a range of $280^{\circ}$ for joint four, $200^{\circ}$ for joint five, and $532^{\circ}$ for joint six. However, to avoid hitting itself, joint five cannot have a range greater than $360^{\circ}$. This limitation generates infeasible regions inside the joint five workspace $\left(O W K_{5}\right)$. The infeasibility in $O W K_{5}$ can be resolved by adjusting $\theta_{1}, \theta_{2}+\theta_{3}$, and $\theta_{4} \cdot{ }^{11}$ The workspaces of joints four and six are feasible when their ranges are larger than $180^{\circ} .^{9}$ With a broad feasible range for $O W K^{m}$, the proposed scheme will first modify the traces in $P W K$ to obtain a feasible collision-free wrist path when possible. Then, based on the new $R_{w}$ and the specified $R_{e}$ on the planned path, new traces in $O W K^{m}$ can be derived and modified if necessary. Consequently, the planned path in Cartesian space can be obtained using these new traces. The algorithm is as follows:

Collision-Free Position Modification Algorithm: Maintain the orientation specification by modifying the positions in the presence of obstacles.

Step 1: Enlarge the radius of $O B S$ by the length $\sqrt{h_{y}^{2}+h_{z}^{2}}$

Step 2: Find the wrist position $p_{w}$ from the path. Derive the corresponding trace of $\underline{p}_{w}$ in $P W K_{1}$, denoted by $t r_{1}$.

Step 3: Obtain $O B S_{1}$. Identify the portions of $t r_{1}$ in $P W K_{1}$ within the CFR and CPR. For the portion within the $C F R$, do the processing in $P W K_{23}$ without considering $O B S$; otherwise, $O B S$ needs to be taken into account.

Step 4: Obtain $O B S_{23}$. Derive the corresponding trace of $p_{w}$ in $P W K_{23}$, denoted by $t r_{23}$. Then modify the infeasible portion of $\operatorname{tr}_{23}$ to be within feasible regions when possible. The modified trace is denoted by ${ }^{n} t r_{23}$. Possible modifications can be to move the infeasible portion to be along the boundary of feasible regions or just to connect the starting and end locations of the infeasible portion with an arbitrary feasible trace. If modification fails, declare that there is no feasible path available and exit. Note that due to the modification of $t r_{23}$ to " $t r_{23}, t r_{1}$ will deviate to a new trace ${ }^{n} t r_{1}$, while $t r_{1}$ and ${ }^{n} t r_{1}$ may correspond to the same set of joint variables. 
Step 5: Find the new wrist orientation ${ }^{n} R_{w}$ corresponding to ${ }^{n} t r_{1}$ and ${ }^{n} t r_{23}$. Derive the new traces $t r^{m}$ in $O W K^{m}$ using ${ }^{w} R_{e}$ derived from ${ }^{n} R_{w}$ and $R_{e}$ on the initial planned path. Check the feasibility of the trace in $O W K_{5}$ and modify it when infeasible and possible. If modification fails, declare that there is no feasible path available and exit.

Step 6: Find the corresponding path in Cartesian space using these five newly derived traces.

\subsubsection{Orientation Modification in the Presence of Obstacles}

In contrast to position modification, orientation modification is developed for cases with the position limitation. With the position limitation, we need to design the tip path to be collision-free in step 1 of the path planning algorithm. The dof in orientation will then be used to modify the traces in the workspaces by maintaining the tip path. Unlike position modification, in orientation modification it is not uncommon for modification to be impossible. With a collision-free tip path, the necessary and sufficient condition for a successful modification is that every point on the path must correspond to at least one feasible wrist position and have feasible orientation(s) in $O W K^{12}$ and all points on the tool need to be collision-free. Because the tool-tip position is fixed, the feasible wrist positions will be on the surface of a sphere that is centered at the tool-tip with the length of the tool as the radius. Thus, to obtain feasible wrist positions, the sphere must intersect $P W K$. In addition, at least one of the intersecting points has to be feasible in PWK and correspond to feasible orientation(s) in OWK that allows the designated tip location to be reached.

To ensure that points on the tool are collisionfree, not only must the wrist be moved away from the obstacle, but also a certain margin must be maintained between the wrist and the obstacle to keep all the points on the tool collision-free. Because two lengths with different orientations are incorporated into the tool, two dof provide the fixed tip and wrist locations to be connected by these two lengths with various combinations of orientations. To avoid checking for all points on these two lengths with different orientations, we propose to enlarge the radius of the obstacle, as in position modification, in the process of determining the margin kept between the wrist and the obstacle. The enlarged radius, however, will depend on the distance between the obstacle and the tool-tip, and it will be a portion of the tool length instead of the entire length. The rea- son that the enlarged radius need not be the entire tool length is because the distance between the tooltip and the obstacle is known in orientation modification; consequently, their geometrical relationship can be analyzed and utilized. Thus less feasible regions are discarded in orientation modification, which now must satisfy more strict constraints than those for position modification. The enlarged radius is defined as follows:

Definition 1. Enlarged Radius (ER): For a fixed tool-tip position, the ER is the minimum distance between the center of the obstacle and the wrist such that all the points on the tool will be collisionfree, no matter what orientations the two lengths of the tool have.

To modify an infeasible modifiable path, we first find a "most infeasible point" (MIP) for the points within the infeasible portion of the planned path. ${ }^{11}$ The MIP is then modified into feasible regions with the position of the MIP maintained by varying its orientation. Among the feasible points with the same position as $M I P$, a "most feasible point" (MFP) is selected to be the modified MIP. The MIP and MFP are defined as follows ${ }^{11}$ :

Definition 2. Most Infeasible Point (MIP): Among those points within the infeasible portion of the planned path, MIP is the point that moves the greatest distance when it is moved into the nearest feasible collision-free location.

Definition 3. Most Feasible Point (MFP): Among those feasible points with the same position as $M I P, M F P$ is the point that moves the greatest distance when it is moved to the nearest infeasible location due to kinematic constraints and obstacles.

The path will now be replanned by interpolating the orientational parts of the starting point, the $M F P$, and the end point of the infeasible portion. If infeasible portions are still present after replanning, they will be identified and the procedure repeated until a feasible path is found. Because the derivation of MIP and MFP implies that a "most infeasible" point is modified to a "most feasible" location, the replanning tends to reduce much of the infeasible portion. Consequently, the number of modifications may decrease. The algorithm for orientation modification is as follows. 
Collision-Free Orientation Modification Algorithm: Maintain the position specification by modifying the orientations in the presence of obstacles.

Step 1: For each point on the path, find the enlarged radius $E R$ for $O B S$ according to the distance between the tool-tip and the center of $O B S$.

Step 2: Using the enlarged obstacle with ER, rotate the tool around the tool-tip to check if each point on the path has intersecting points with $P W K$ where the tool is collision-free. If the result is negative, then declare infeasible inputs and exit.

Step 3: For each point, check if at least one of the intersecting points derived in step 2 is feasible in $P W K$ and corresponds to feasible orientation(s) in OWK that allows the designated tip location to be reached. The checking process is divided into separate procedures for the $C F R$ and $C P R$, similar to those described in the previous section for position modification. However, the checking process here deals with the obstacle of the original radius, because the checking for a collision-free tool has been performed. If the result is negative, then declare infeasible inputs and exit.

Step 4: Identify the infeasible portion of the planned path. Then find the MIP and its corresponding $M F P$.

Step 5: Replan the path by interpolating the orientational parts of the starting point, the MFP, and the end point of the infeasible portion.

Step 6: If the replanned portion is feasible, declare the modification successful and exit. Otherwise, go back to step 4 .

\section{SIMULATIONS}

To demonstrate the proposed scheme, we used it to plan separate robot paths satisfying (a) an orientation specification and (b) a position specification. The joint ranges chosen for the robot manipulator were similar to those of industrial robot manipulators. The kinematic specifications for the robot manipulator were set as $a_{2}=0.6 \mathrm{~m}, d_{1}=1.2 \mathrm{~m}, d_{4}=$ $0.4 \mathrm{~m}, h_{y}=0.05 \mathrm{~m}$, and $h_{z}=0.05 \mathrm{~m}$. The ranges of joint one to joint six were $\left(-60^{\circ}, 135^{\circ}\right),\left(-135^{\circ}, 135^{\circ}\right)$, $\left(-135^{\circ}, 60^{\circ}\right),\left(-60^{\circ}, 200^{\circ}\right),\left(-50^{\circ}, 150^{\circ}\right)$, and $\left(-200^{\circ}\right.$, $200^{\circ}$ ), respectively. The obstacle was defined to be of radius $0.08 \mathrm{~m}$.

To plan a path satisfying an orientation specification, first we designed an initial path that allowed the tool-tip of the robot to follow a line with a fixed orientation. Modifications were then performed to

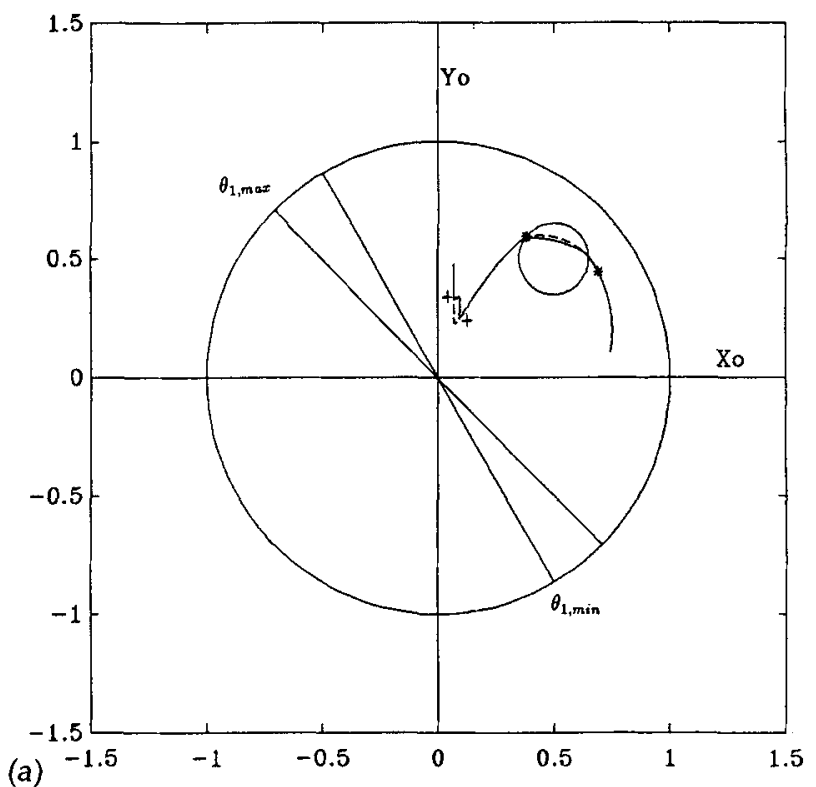

Figure 6. Traces in the positional workspace. (a) Joint one workspace.

maintain the fixed orientation by adjusting the positions on the line. The obstacle was assumed to be centered at $(-0.5,0.5,1.5)$. Based on the discussions in section 2, the initial path was mapped into $P W K$ and $O W K$; the traces corresponding to this path are shown as dotted lines in Figures 6 and 7. Following the collision-free position modification algorithm given in the previous section, we enlarged

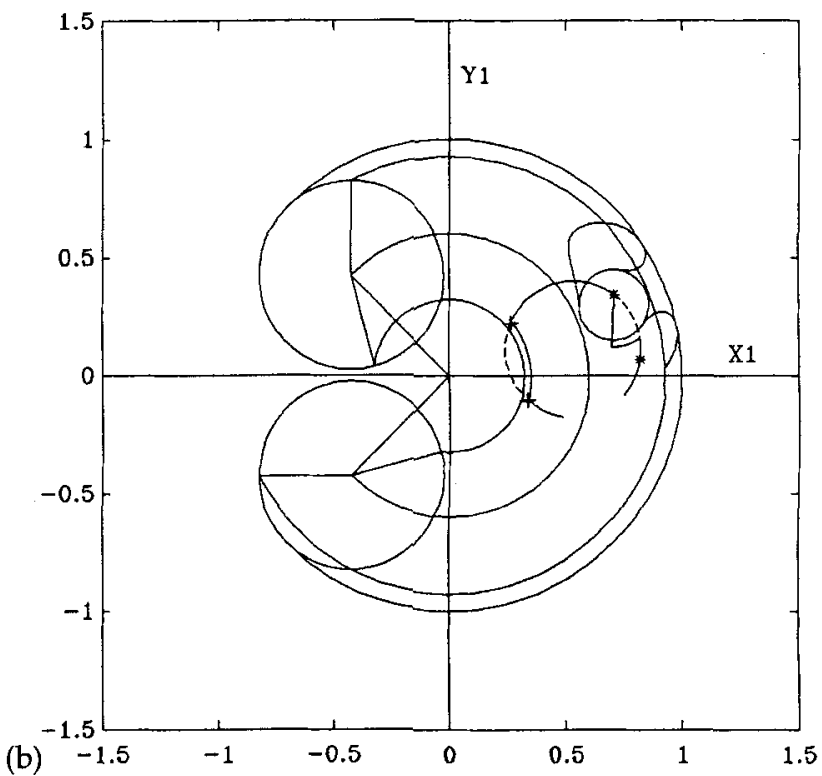

Figure 6. Trace in the positional workspace. (b) Joints two and three workspace. 


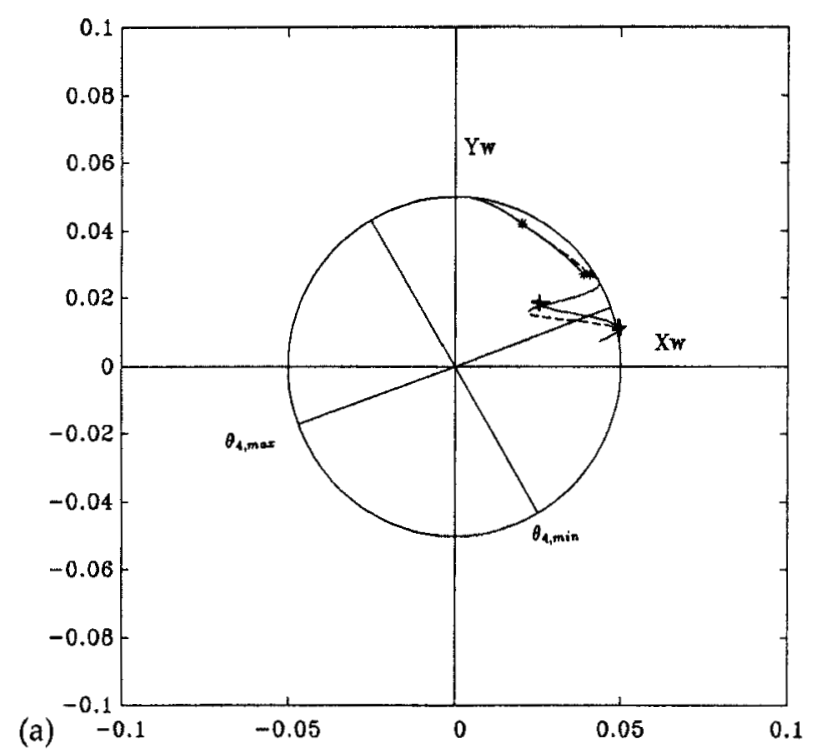

Figure 7. Traces in the orientational workspace. (a) Joint four workspace.

the radius of the obstacle to $0.15 \mathrm{~m}$. In Figure $6 \mathrm{a}$, the trace, which is the mapping of the designed path on $P W K_{1}$, falls within both CFR and CPR. The portion of the trace that falls within the CPR in $P W K_{1}$ and its corresponding mapping in $P W K_{23}$ are shown between two asterisks $(*)$. Therefore, the portion between the two asterisks had to be modified in the presence of $O B S_{23}$ and the rest of the trade did not. Modifications corresponding to these two regions

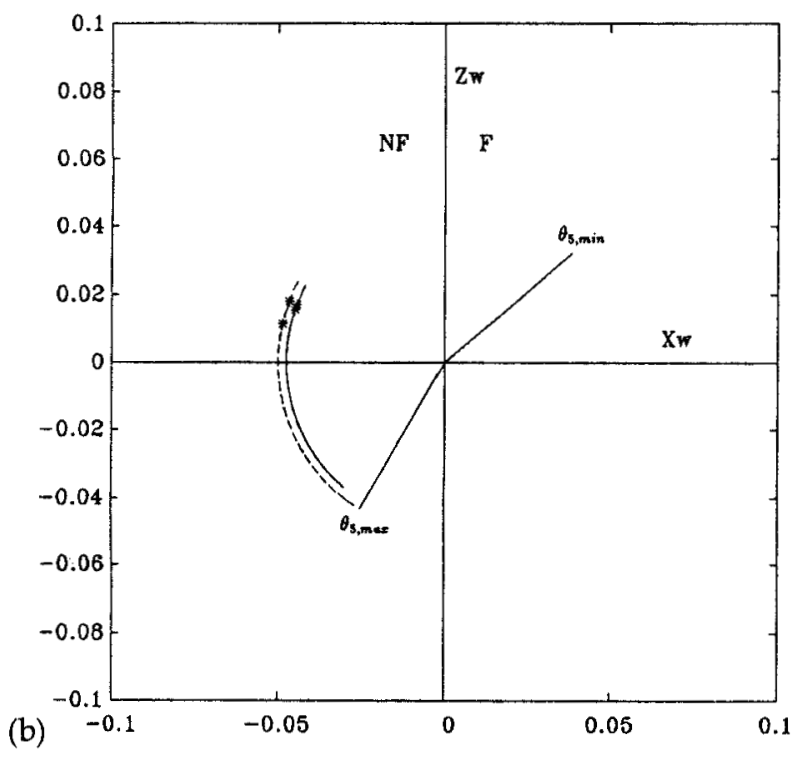

Figure 7. Traces in the orientational workspace. (b) Joint five workspace.

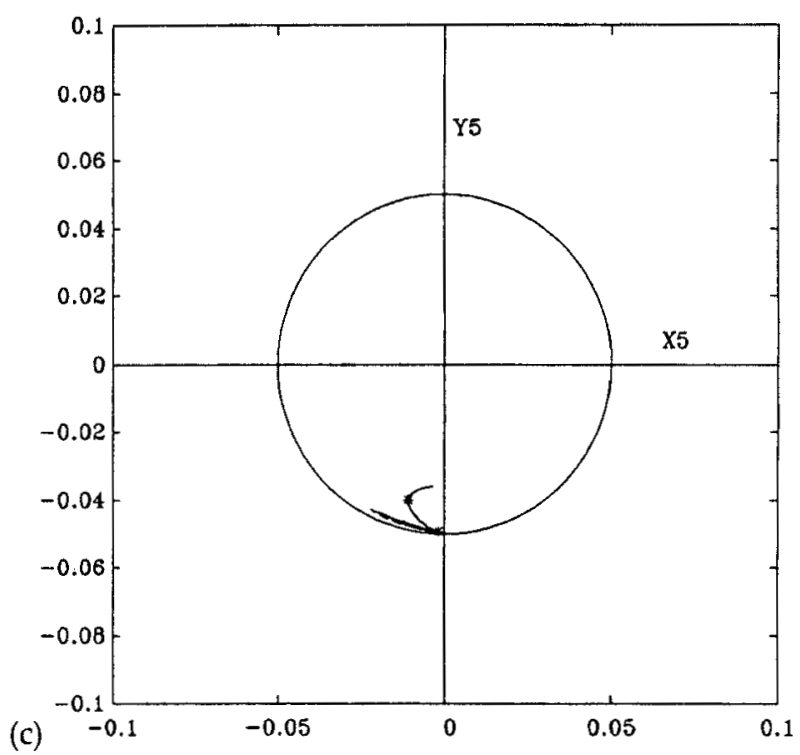

Figure 7. Traces in the orientational workspace. (c) Joint six workspace.

are shown in Figures $8(\mathrm{~b})$ and $9(\mathrm{~b})$. New traces in $P W K_{1}$ due to the modifications shown in Figures $8(b)$ and $9(b)$ are shown in Figures 8(a) and 9(a). The resulting modified traces in $P W K_{1}$ and $P W K_{23}$ are shown as solid lines in Figures 6(a) and 6(b), respectively. In Figures 6-9, the modified traces corresponding to the $C F R$ are those between two plus signs $(+)$. Then, by utilizing the new wrist coordinates obtained from the modified traces in PWK along with the specified orientation of the initial path, we obtained new traces in $O W K^{m}$; they are shown as solid lines in Figures 7(a)-(c). These traces were feasible and did not need modification. The resulting modified tip and wrist paths are shown in Figure 10, where they overlay the original paths. The scheme successfully modified the original infeasible line into a feasible one while maintaining the original orientation.

We performed a second simulation to plan a path satisfying a position specification. First, we designed an initial path that allowed the robot's tooltip to follow a collision-free straight line from $(0.87$, $0.06,1.31)$ to $(0.42,0.21,1.53)$ with a fixed orientation. The obstacle was assumed to be centered at $(0.68,0.24,1.46)$. The planned path was found to be infeasible and modifiable. Modification was then performed to maintain the specified positions by adjusting the orientation on the line. The original and modified tip and wrist paths are shown in Figure 11. The modification scheme maintained the original straight-line path while modifying its orientation. 


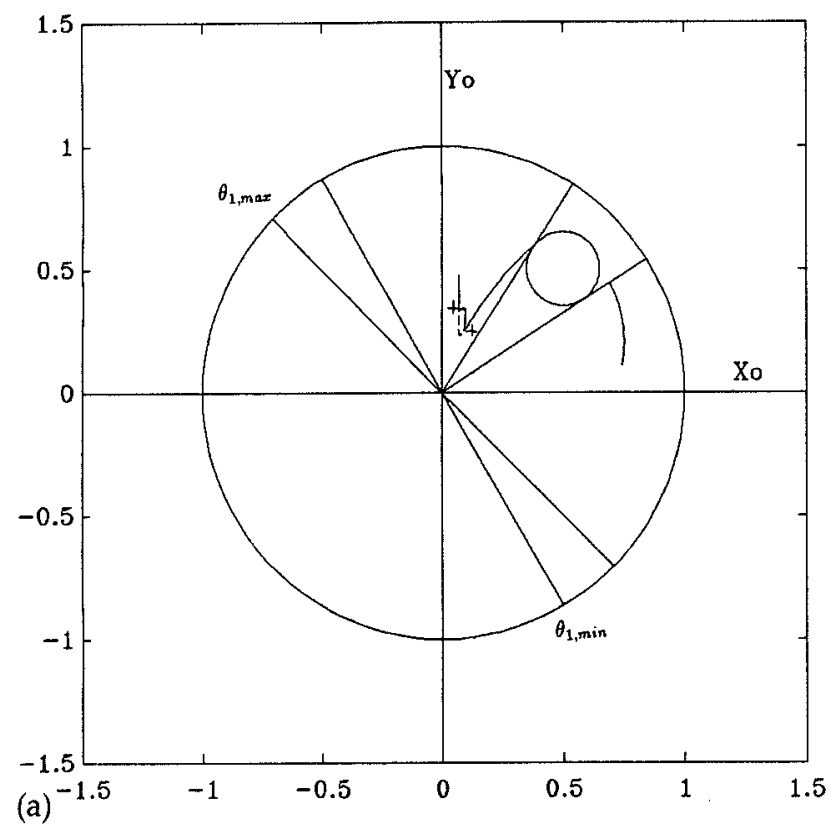

Figure 8. Modification for $C F R$ in the positional workspace. (a) Joint one workspace.

Consequently, the original straight-line wrist path was modified to be a curved one.

\section{DISCUSSION AND CONCLUSION}

Our path planning scheme efficiently designs a kinematically feasible path in the presence of obsta-

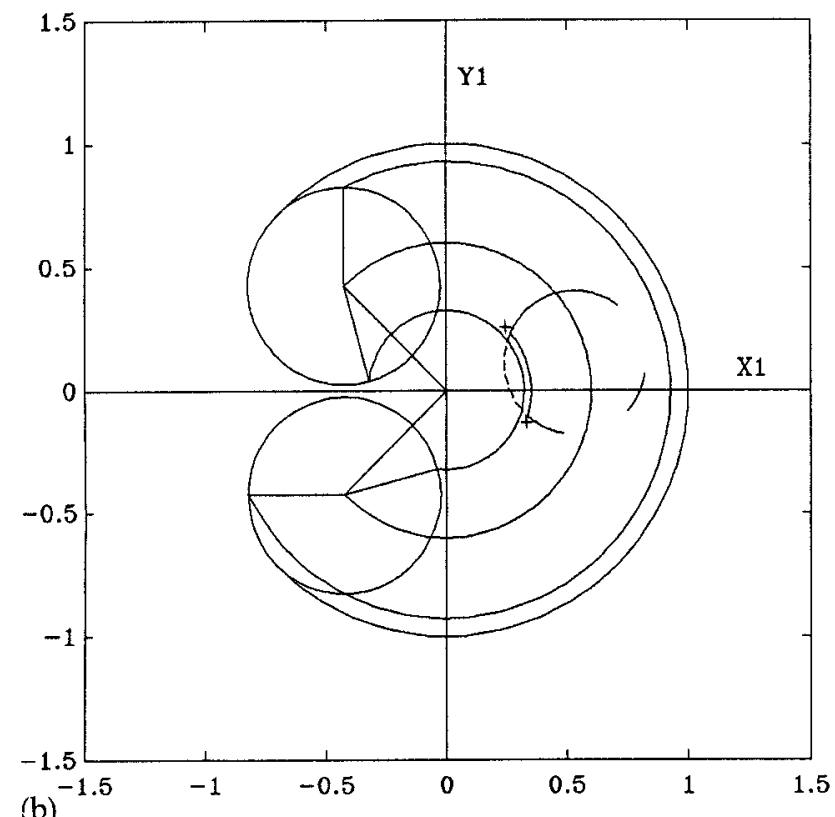

Figure 8. Modification for $C F R$ in the positional workspace. (b) Joints two and three workspace.

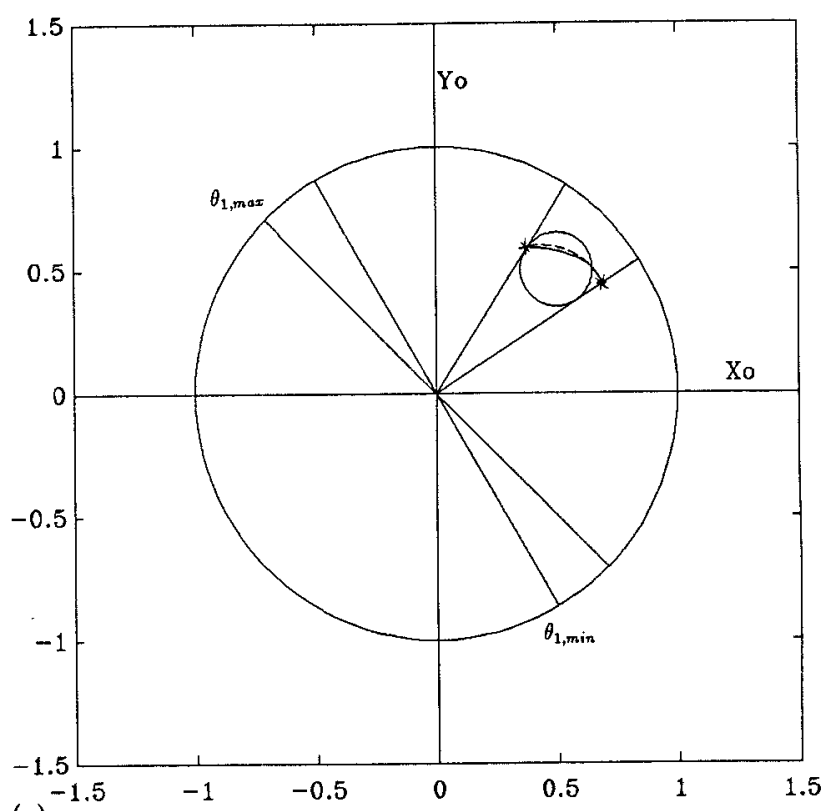

(a)

Figure 9. Modification for $C P R$ in the positional workspace. (a) Joint one workspace.

cles based on task requirements and can be extended to general wrist-partitioned types of robot manipulators and arbitrarily shaped obstacles. In addition, a merit of the proposed path planning scheme is that task requirements are incorporated into the planning, which is not well explored in previous works. A quantitative analysis on the issue of

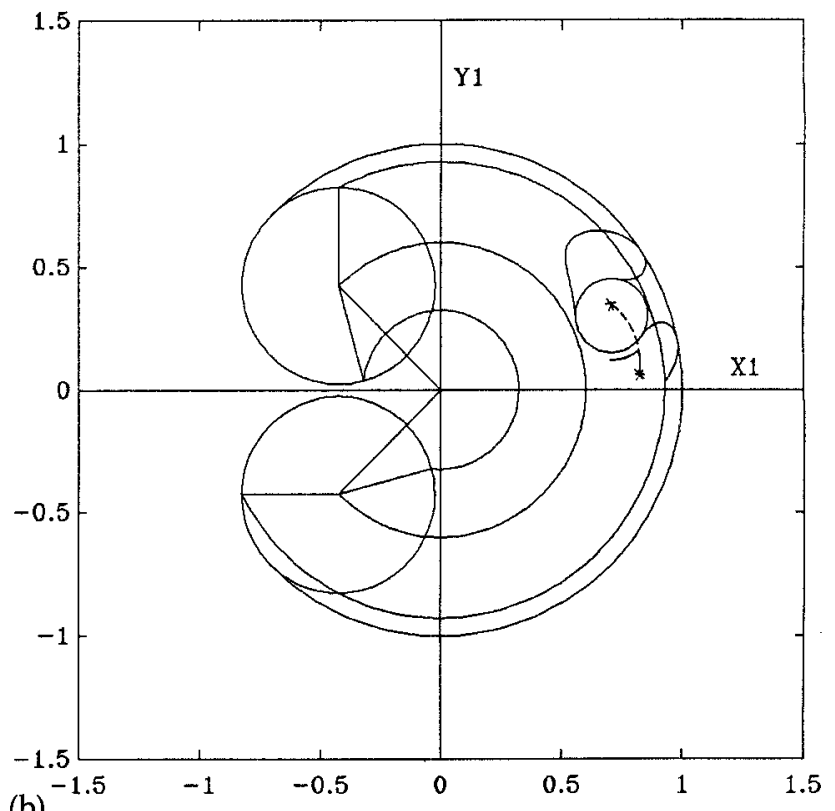

(b)

Figure 9. Modification for $C P R$ in the positional workspace. (b) Joints two and three workspace. 


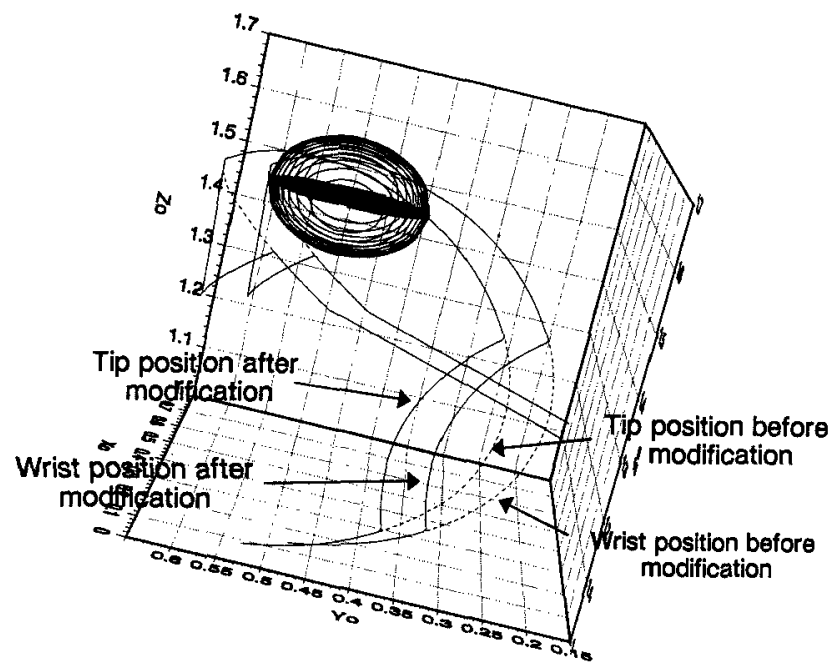

Figure 10. Tip and wrist paths before and after position modification.

efficiency is not discussed in this article, such as complexity analysis or running time comparison with other approaches. Nevertheless, from the qualitative point of view, the proposed scheme promises to be efficient in both obstacle formulation and path planning. In obstacle formulation, an arbitrarily shaped obstacle is approximated by several spherical obstacles of equal or different radii. Thus simple and similar geometrical analysis can be imposed upon the same shape of obstacles of different sizes. For comparison, a close approximation of the obstacle can be achieved at the expense of computation time by applying the cell decomposition approach, or tree-based technique, etc. ${ }^{5,13}$ In turn, with com-

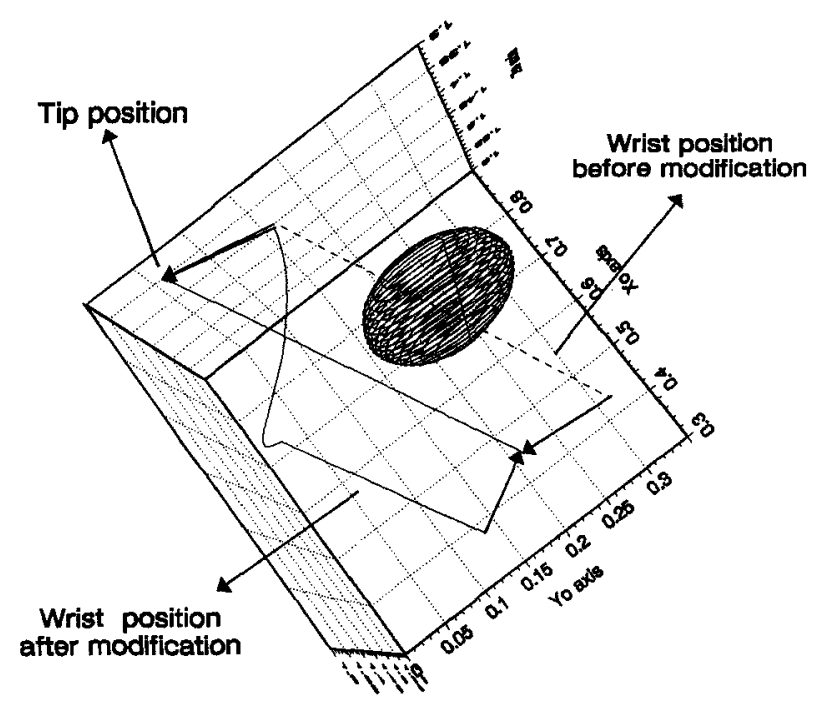

Figure 11. Tip and wrist paths before and after orientation modification. plicated obstacle formulation in the robot workspace, the search for feasible paths is time consuming. Thus, in a sense, with the proposed obstacle formulation the efficiency in obstacle description and path planning improves at the expense of losing feasible regions in the process of approximation. In addition, we utilize modification strategies to assist in this task-dependent path planning, which involves a smaller local search space, and consequently demands less running time.

It can be seen that when an irregularly shaped obstacle is represented by several spheres, some of the feasible regions will be discarded in the approximation. It is even more noticeable for those shapes that may not be suitable to be approximated by spheres as the basic element, e.g., a long rod. One resolution will be a trade-off between losing more feasible regions and spending more computation time in using more spheres for approximation. Another resolution as mentioned in section 3.2 may be that obstacle shapes other than a sphere be used as the basic element in constructing various shapes of obstacles. The choice of which shape to use involves another trade-off between a better representation for geometrical analysis and a better element for constructing the target obstacles.

One of our future objectives is to resolve this difficulty by finding more appropriate geometrical representations for obstacles of various shapes. In other words, the representation should be time-efficient and result in losing less feasible region. One possible proposal is to perform obstacle formulation according to the structure of the given robot and geometrical properties of the links. 8,13 In some sense, obstacle formulation will be tackled on the basis of joints; in contrast, in this article the obstacle formulation is in Cartesian space. Note that certain feasible regions cannot be well utilized due to the structure of the links, e.g., the feasible regions in the zig-zag edges of an obstacle. Therefore, obstacle formulation according to the structure and movements of the links may lead to losing less utilizable feasible regions in the approximation and deserves further exploration.

This work was supported in part by the National Science Council, Taiwan, R.O.C., under grant NSC 810422-E-009-09.

\section{REFERENCES}

1. E. G. Gilbert and D. W. Johnson, "Distance functions and their application to robot path planning in the 
presence of obstacles," IEEE J. Rob. Autom., 1(1), 2130, 1985.

2. J. M. Hollerbach, "Dynamic scaling of manipulator trajectories," J. Dyn. Syst. Meas. Control, Trans. ASME, 106, 102-106, 1984.

3. R. P. Paul, "Manipulator Cartesian path control," IEEE Trans. Syst. Man Cybern., 9(11), 702-711, 1979.

4. R. H. Taylor, "Planning and execution of straight line manipulator trajectories," IBM J. Res. Dev., 23(4), 424-436, 1979.

5. T. Lozano-Perez, "A simple motion-planning algorithm for general robot manipulators," IEEE J. Rob. Autom., 3(3), 224-238, 1987.

6. K. K. Gupta, "Fast collision avoidance for manipulator arms: A sequential search strategy," IEEE Trans. Rob. Autom., 6(5), 522-532, 1990.

7. C. S. G. Lee and M. Ziegler, "A geometric approach in solving the inverse kinematics of PUMA robots," IEEE Trans. Aerosp. Electron. Syst., 20(6), 695-706, 1984.
8. V. Lumelsky, "Effect of kinematics on motion planning for planar robot arms moving amidst unknown obstacles," IEEE J. Rob. Autom. 3(3), 207-223, 1987.

9. K. Y. Young and C. H. Wu, "Path feasibility and modification," J. Rob. Syst., 9(5), 613-633, 1992.

10. F. L. Litvin, Z. Yi, V. P. Castelli, and C. Innocenti, "Singularities, configurations, and displacement functions for manipulators," Int. J. Rob. Res., 5(2), 52$65,1986$.

11. K. C. Shao and K. Y. Young, "Path feasibility and modification based on the PUMA 560 workspace analysis," J. Mech. Des., Trans. ASME, 116(1), 36-43, 1994.

12. C. C. Jou, "On the orientational feasibility of robot manipulators," Int. Conf. Automation, Robotics, and Computer Vision, 1990, pp. 524-528.

13. J.-C. Latombe, Robot Motion Planning, Kluwer Academic Publishers, New York, 1991. 